Check for updates

Cite this: RSC Adv., 2019, 9, 24154

\title{
Novel biodegradable and non-fouling systems for controlled-release based on poly( $\varepsilon$-caprolactone)/ Quercetin blends and biomimetic bacterial S-layer coatings $\dagger$
}

\author{
Eva Sanchez-Rexach, (DD $\ddagger^{* a}$ Jagoba Iturri, (D) $\ddagger^{\mathrm{b}}$ Jorge Fernandez, (D) a \\ Emilio Meaurio, (D) Jose-Luis Toca-Herrera ${ }^{b}$ and Jose-Ramon Sarasua ${ }^{a}$
}

\begin{abstract}
Quercetin is a strong antioxidant with low bioavailability due to its high crystallinity. A further drawback is that Quercetin has potentially toxic effects at high concentrations. To improve this low water solubility, as well as control the concentration of the flavonoid in the body, Quercetin is incorporated into a polymeric matrix to form an amorphous solid dispersion (ASD) stable enough to resist the recrystallization of the drug. For this purpose, miscible poly $(\varepsilon$-caprolactone) (PCL) and Quercetin (Q) blends are prepared, provided that they have complementary interacting groups. For compositions in which the flavonoid remains in an amorphous state thanks to the interactions with polymer chains, various $P C L / Q$ drug release platforms are fabricated: micrometric films by solvent casting, nanometric films by spin coating, and nanofibers by electrospinning. Then, the potential use of bacterial S-layer proteins as release-preventive membranes is tested on PCL-Quercetin blends, due to their ability to construct a biomimetic coating including nanometric pores. For all the platforms, the SbpA coating can maintain a stable release under the toxicity level of Quercetin. Accordingly, a PCL/Q system with an Slayer coating allows the design of versatile bioavailable Quercetin eluting devices that prevent toxicity

and biofouling issues.
\end{abstract}

Received 11th June 2019

Accepted 27th July 2019

DOI: $10.1039 / c 9 r a 04398 e$

rsc.li/rsc-advances

\section{Introduction}

Flavonoids are polyphenolic compounds synthesized by plants, which present inhibitory activity against organisms that cause plant diseases. ${ }^{1}$ These compounds have recently gained interest, owing to the potential health benefits arising from their anti-oxidant activity. In the group of flavonoid-rich natural foods one could mention onions, tea, strawberries, apples, grapes, parsley, and many spices, among others. It has been found that the functional hydroxyl groups found in flavonoids have the ability to scavenge free radicals, as well as to induce human protective enzyme systems. ${ }^{2}$ Numerous investigations have also suggested protective effects of flavonoids against

${ }^{a}$ Department of Mining-Metallurgy Engineering and Materials Science, University of the Basque Country UPV/EHU, Plaza Ingeniero Torres Quevedo 1, Bilbao 48013, Spain.E-mail: evagloria.sanchez@ehu.eus

${ }^{b}$ Institute for Biophysics, Department of Nanobiotechnology, University of Natural Resources and Life Sciences (BOKU), Muthgasse 11 (Simon Zeisel Haus), Vienna 1190, Austria

$\dagger$ Electronic supplementary information (ESI) available: Correlation between film thickness and spinning speed illustrated with AFM images, DSC curves, ATR-FTIR spectra, contact angle measurements, and AFM height micrographs showing the topographical features for various PCL-Quercetin systems. See DOI: 10.1039/c9ra04398e

\$ These authors contributed equally to this work. many infectious and degenerative illnesses such as cardiovascular diseases, and other age-related disorders. ${ }^{3}$

In particular, Quercetin $(\mathrm{Q})$ is a well-known flavonoid with anti-oxidant and anti-inflammatory properties. As a drawback, it easily oxidizes (to form a quinone) in aqueous aerobic environments such as body fluids. ${ }^{4}$ Quercetin is especially crystalline, meaning that the energy required to break the intermolecular interactions within its natural crystalline network is high enough not to be effectively dissolved by water, which causes its poor absorption in the gastrointestinal tract. ${ }^{5}$ Furthermore, the bioavailability of Quercetin through oral dosage has been reported to be only around 2\%. ${ }^{6}$ Another disadvantage is that, depending on the dose, Quercetin might shift from anti-oxidant to pro-oxidant effects and hence induce the generation of free radicals, responsible for mutagenic and cytotoxic effects. ${ }^{7}$ A good strategy to enhance the bioavailability and therapeutic efficiency of Quercetin is the formulation of amorphous solid dispersions (ASDs). In these ASDs, polymer chains (i.e. polyesters) act as crystallization inhibitor and thus preserve the drug in its amorphous form. Although less stable than its crystalline version, the amorphous form of Quercetin can certainly be stabilized by strong physical interactions (i.e. hydrogen bonds) with the polymeric matrix. In this regard, poly(e-caprolactone) (PCL) is one of the most promising 
biodegradable polyesters widely used for long-term biomedical applications known its slow degradation can last from several months to years. ${ }^{8}$ Considering the chemical structures of the two species mentioned (PCL and Quercetin), a miscible polymer-drug system in the form of ASDs can be envisaged. In addition, hydrogen bond formation between the hydroxyl groups of Quercetin and the carbonyl groups of PCL can be expected, which would overcome the strong auto-association interactions occurring between the Quercetin molecules in the crystalline phase. ${ }^{9}$ The combination of the unique properties of anti-oxidant Quercetin molecules with the versatility of a biopolymer such as the PCL, can lead to obtaining controlled release of the flavonoid.

As aforementioned, and despite the beneficial health effects brought by Quercetin, this flavonoid is potentially cytotoxic at high doses. ${ }^{\mathbf{1 0}}$ In order to control the amount released from the PCL matrix the usage of an outermost membrane or any type of (semi)permeable thin coating would be desirable. The answer to such a critical issue was found in a natural system: bacterial surface S-layer proteins. ${ }^{11}$ Such a polycrystalline coating of only a few nanometers thickness is formed by an array of glycoproteins following diverse crystalline symmetries (oblique, squared, hexagonal...). S-Layers are found in the outermost envelope of prokaryotes, and represent not only the simplest biological membrane but also a highly efficient protective membrane with proven anti-fouling activity. ${ }^{12,13}$ From the bioengineering point of view, it is highly interesting that isolated S-layer protein sub-units have the ability to self-assemble, through the so-called recrystallization process, on a broad number of organic and inorganic supports. ${ }^{\mathbf{1 4 , 1 5}}$ This is the case of SbpA from Lysinibacillus sphaericus CCM2177, which presents a square (p4) lattice symmetry, a system that has quite extensively been characterized in literature so far. ${ }^{\mathbf{1 6 - 1 8}}$ Because of the large number of nanometric pores found along the S-layer structure, it can be hypothesized that the release of the drug from the polymeric matrix can be still conducted although to a lower rate.

Hence, in this work, the synthesis of PCL/Q blends as miscible ASDs in both 2D (films) and 3D (mats) polymeric platforms is presented for the first time. The miscibility and topography of the resulting specimens was characterized by different techniques (DSC, ATR-FTIR, AFM, and XRD) and, the respective drug release kinetics could be evaluated both in the presence and in the absence of an outermost SbpA S-layer. The focus was specifically set on the $90 / 10$ and $80 / 20$ PCL/Q compositions, where the recrystallization of the Quercetin is successfully inhibited. The initial release of Quercetin from micrometric $(\sim 200 \mu \mathrm{m})$ and thin nanometric films $(\sim 200 \mathrm{~nm})$ was studied by means of either UV-Vis spectroscopy or quartz crystal microbalance with dissipation monitoring (QCM-D), respectively. Quercetin was also encapsulated in PCL nanofibers to study the effect of the 3D structure on the release. The results confirmed the possibility of constructing different PCL/ Q platforms from which Quercetin can be successfully released, thus making this system suitable for different applications. In addition, the self-assembly of SbpA on the PCL/Q blends was proved by means of QCM-D, which allowed a real time characterization of the process, and complementary AFM topography imaging to analyze the lattice parameters, the size of the crystal domains as well as the stability of the formed supramolecular structure. Contact angle measurements were also performed to assess the SbpA coating on micrometric films. The presence of the biomimetic membrane is shown to critically impact the release of the drug, showing an overall decrease of around the $30 \%$, as well as the resistance of PCL/Q samples against non-specific adhesion of foulants, based on the HUVEC cell proliferation studies performed.

The novelty of this work, resides in the use of a biologicallyderived supramolecular structure as a release controller of a natural anti-oxidant from a biopolymer for a sustained activity of the Quercetin for therapeutic applications. Furthermore, Slayer coating has demonstrated antifouling properties. Consequently, this newly developed PCL-Quercetin system with a SbpA S-layer on top, can be useful for making biodegradable drug-eluting devices with the extra ability to prevent the adhesion of bacteria to the surface, thus avoiding the undesirable biofilm formation that implies infections on implants.

\section{Experimental}

\section{Materials}

Polycaprolactone (PURASORB ${ }^{\circledR}$ PC12 trade name) with an average molecular weight $\left(M_{\mathrm{w}}\right)$ of $1.3 \times 10^{5} \mathrm{~g} \mathrm{~mol}^{-1}$ and $M_{\mathrm{w}} / M_{\mathrm{n}}$ $=1.76$ (as determined by GPC) was obtained from Purac Biochem (The Netherlands). Quercetin (purity $\geq 95 \%$ ), formic acid, and phosphate buffered saline tablets (PBS) 0.1 M, pH 7.4, were supplied by Sigma-Aldrich (Spain). Tetrahydrofuran (THF) and 2,2,2-trifluoroethanol (TFE) were purchased from Labbox (Spain).

Bacterial cell-surface layer protein, $\operatorname{SbpA}\left(M_{\mathrm{w}}=120 \mathrm{kDa}\right)$ was isolated and purified from L. sphaericus CCM 2177 following standard protocols described elsewhere. ${ }^{19}$ Protein recrystallization buffer was pre-pared with $0.5 \mathrm{mM}$ Trizma base (Sigma-

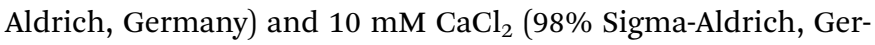
many) and adjusted to $\mathrm{pH} 8$.

\section{Blend preparation}

Micro- and nano-metric films. Preparation of $200 \mu \mathrm{m}$ thick films proceeded from casting of PCL/Q solutions in $2 \mathrm{wt} \%$ tetrahydrofuran (THF), kept overnight at room temperature into $40 \mathrm{~mm}$ diameter glass Petri dishes. In turn, nanometric films were obtained by controlled spin coating (Schaefer Tech, Germany) of PCL or PCL/Q solutions (10 $\mathrm{mg} \mathrm{mL}^{-1}$ ) onto either borosilicate glass substrates or QCM-D sensors. The spin coater operated at $3600 \mathrm{rpm}$, thus resulting in $200 \mathrm{~nm}$ thick films. In order to establish the correlation between film thickness and rotating speeds, 2 drops of PCL solution were deposited on spinning glass substrates under pre-defined speed values: 500, 1000,2000 or $3600 \mathrm{rpm}$. Thus, the thickness of these films could be evaluated by simply removing a stripe of the material (acetone sweeping) and subsequently quantifying the height of the step generated by means of Atomic Force Microscopy (AFM). The correlation between film thickness and spinning speed, as 
well as the spherulitic morphology for thin films of different dimensions, are shown in the Fig. S1 of the ESI. $\dagger$

Scaffolds. For the production of the porous scaffolds, 90/10 PCL/Q solutions (4.5 vs. $0.5 \mathrm{~g}$, respectively) were prepared using a mixture of TFE $(11 \mathrm{~mL})$ and formic acid (FA, $4 \mathrm{~mL})$, added to increase the conductivity. The resulting mixture was subsequently vortexed to ensure proper mixing. Afterwards, polymer electrospinning was performed at room temperature $\left(21 \pm 2{ }^{\circ} \mathrm{C}\right)$ with controlled humidity $(\sim 40 \%)$ in a Ne200 Single Nozzle Electrospinning Machine (Inovenso, MA, USA). The tunable high-voltage power supply was connected to the tip of the needle (positive lead) and attached to the collector (negative lead) with an alligator clip. The needle-to-collector distance was of $20 \mathrm{~cm}$ and the polymer solutions were ejected using a syringe pump at an adjusted flow rate. Polymer mats were spun directly onto a plate-shaped collector for 1 hour to achieve rectangular samples with a thickness of approximately $125 \mu \mathrm{m}$. Steady conditions were achieved at a flow rate of $1.0 \mathrm{~mL} \mathrm{~h}^{-1}$ and 19.9 $\mathrm{kV}$ of voltage.

\section{S-Layer coating}

After isolation, the protein solution was centrifuged at $5000 \mathrm{rpm}$ for $5 \mathrm{~min}$ in order to separate the S-protein monomers from selfassembly products and was stored at $4{ }^{\circ} \mathrm{C}$. Then, before each experiment, supernatant solution was diluted using the appropriate amount of recrystallization buffer (see description above) to a final concentration of $0.1 \mathrm{mg} \mathrm{mL} \mathrm{m}^{-1}(c a .85 \mu \mathrm{m})$. The S-layer recrystallization on micrometric films and scaffolds of PCLQuercetin blends was achieved through the incubation at $4{ }^{\circ} \mathrm{C}$ of square samples $\left(1 \mathrm{~cm}^{2}\right)$ into the dissolution of SbpA for 2 hours. For nanometric films, the S-protein assembly was followed in situ by means of QCM-D experiments described below.

\section{Sample characterization}

FE-SEM analysis. Electrospun mats were examined by means of Field Emission Scanning Electron Microscopy (FE-SEM). PCL-Quercetin scaffolds were first sputter-coated with a thin layer of gold $(\sim 15 \mathrm{~nm})$ in an Emitech K550X and observed in a Hitachi SEM (Hitachi S-4800, Tokyo, Japan). The voltage used was $10.0 \mathrm{kV}$ and the working distance was $7.0-9.0 \mathrm{~mm}$ with a magnification of $1000 \times, 5000 \times$ and $20000 \times$. In order to assess the average diameter, over 100 individual fibers were measured by Image J software using SEM images from at least five different sections of each sample.

Differential scanning calorimetry (DSC). Thermal analyses were conducted on a Modulated DSC Q200 (TA Instruments, DE, USA). All scans were carried out in hermetic aluminium pans under a nitrogen atmosphere for sample weights between 5 and $10 \mathrm{mg}$. Prior to these measurements, an isotherm at a temperature of $110{ }^{\circ} \mathrm{C}$ was performed during $30 \mathrm{~min}$ to eliminate residual water content of Quercetin. For the purpose of studying glass transition temperatures, two consecutive scans were performed from $-80^{\circ} \mathrm{C}$ to $340^{\circ} \mathrm{C}$ with a scan rate of $20^{\circ} \mathrm{C} \mathrm{min}^{-1}$, which ensured complete melting of the sample. The first scan allowed measuring the blend melting temperatures $\left(T_{\mathrm{m}}\right)$, while glass transition temperatures $\left(T_{\mathrm{g}}\right)$ were obtained from the second scan, as midpoint of the specific heat increment. The results obtained are described in the ESI (Fig. S2†).

Attenuated total reflection Fourier transform infrared spectroscopy (ATR-FTIR). ATR-FTIR spectra of the PCL/Q films were recorded on a Thermo Nicolet AVATAR 370 Fourier transform infrared spectrophotometer (Thermo Fisher Scientific, MA, USA) with a mounted PIKE MIRacle ATR accessory containing diamond crystal. Spectra were taken with a resolution of $4 \mathrm{~cm}^{-1}$ and were averaged over 32 scans in the $4000-525 \mathrm{~cm}^{-1}$ range. Prior to performing the spectra of the PCL/Q samples with the ATR-FTIR, they were melted at a temperature of $330{ }^{\circ} \mathrm{C}$ for $5 \mathrm{~min}$, and subsequently allowed to cool to room temperature. The obtained spectra are shown in the Fig. S3 of the ESI. $\dagger$

X-ray diffraction (XRD). X-ray powder diffraction patterns were collected by using a Philips X'pert PRO automatic diffractometer operating at $40 \mathrm{kV}$ and $40 \mathrm{~mA}$, in theta-theta configuration, secondary monochromator with $\mathrm{Cu}-\mathrm{K} \alpha$ radiation $(\lambda=1.5418 \AA)$ and a PIXcel solid state detector (active length in $2 \theta 3.347^{\circ}$ ). Data were collected from 5 to $50^{\circ} 2 \theta$ (step size 0.026 and time per step $=700 \mathrm{~s}$ ) at room temperature. A fixed divergence and anti-scattering slit giving a constant volume of sample illumination was used.

Topographical analysis by means of AFM. For the topographical analysis of PCL-derivative micrometric films, an AFM instrument Nanowizard I (JPK Instruments, Germany) was operated in air, using contact imaging mode at constant loading forces and rates. Cantilevers were calibrated before each experiment by means of thermal tune method. Silicon-nitride probes (DNP-10, Bruker, USA) with a nominal spring constant of $0.12 \mathrm{~N} \mathrm{~m}^{-1}$ were used in the experiments. All images were processed by JPK data analysis software.

In order to extract topographical information of S-layer coated samples, a Nanoscope V multimode AFM (Veeco, Santa Barbara, USA) was employed. Before use, the fluid cell was washed overnight with $2 \%$ SDS, rinsed thoroughly with ultrapure water, and dried with nitrogen. Topography images were recorded in tapping mode, at $1 \mathrm{~Hz}$ in $100 \mathrm{mM} \mathrm{NaCl}$ aqueous solution and at room temperature. The final setpoint was carefully controlled to avoid coating damaging. Silicon nitride $\left(\mathrm{Si}_{3} \mathrm{~N}_{4}\right)$ cantilevers of $0.32 \mathrm{~N} \mathrm{~m}^{-1}$ with sharpened tips (DNPS, Veeco) and gold-coated reflective back sides were cleaned in ethanol before use.

Contact angle. Water contact angle was measured using a DSA-100 drop shape analyzer (Krüss, Germany), and $2 \mu \mathrm{L}$ water droplets. The water droplet images were analyzed using a circular fitting method to obtain the contact angles on the different surfaces. Measurements were made at different positions in each surface for averaged results included in the ESI (Fig. S4†).

\section{In vitro release studies and release kinetics}

As stated above, Quercetin is highly reactive upon exposure to atmospheric or aqueous oxygen, which induces quinone formation. The oxidative degradation reduces the pharmacological efficiency of this anti-oxidant and, in addition, makes 
analytical determination more difficult. ${ }^{20}$ To avoid the oxidation of the Quercetin, the PBS buffer solution used for release experiments was degassed throughout the entire assay by nitrogen recirculation. Such a degassing could not be applied to QCM-D experiments because of chamber design reasons. Instead, a thorough pre-rinse with degassed PBS and incubations in stopped flow conditions ensured system stability over the observation time, taking into account this is a closed system and also the small volume involved. Hence, attending to the type of sample (films, mats) being characterized the following techniques were employed.

Micrometric films and scaffolds (UV-Vis). UV-Vis absorption spectra were recorded over wavelengths ranging from 190 to $500 \mathrm{~nm}$ using a Lambda 265 UV/Vis spectrophotometer (PerkinElmer, Spain). Prior to release experiments, two standard curves with known concentrations of Quercetin were built. On one hand, Quercetin dissolved in tetrahydrofuran with a $\lambda_{\max }$ at $370 \mathrm{~nm}$ was used to measure the initial and the final content of Quercetin in the samples. On the other hand, a second calibration curve was prepared measuring the absorbance of the Quercetin in PBS buffer solution at $376 \mathrm{~nm}$. Experiments were carried out at concentrations lower than $25 \mathrm{ppm}$ because of both solubility issues at higher concentrations, ${ }^{21}$ and maintenance of toxicity-free levels. ${ }^{10}$

Then, PCL/Q films obtained by solvent casting, as well as scaffolds $125 \mu \mathrm{m}$ thick, were immersed in $50 \mathrm{~mL}$ of $0.1 \mathrm{M}$ nitrogen-treated PBS at $37^{\circ} \mathrm{C}(\mathrm{pH}$ 7.4). Sample size in both cases was kept constant $\left(1 \mathrm{~cm}^{2}\right)$ because of comparative purposes. At fixed time intervals, $1 \mathrm{~mL}$ aliquots were extracted and replaced with fresh degassed PBS at $37{ }^{\circ} \mathrm{C}$. Quercetin concentration in solution was determined by UV spectroscopy at a wavelength of $376 \mathrm{~nm}$ (attributed to the B-ring catechol structure), and by using the corresponding calibration curve.

Nanometric films (QCM-D). QCM-D experiments were performed in a Q-Sense E4 instrument (Q-Sense, Biolin Scientific $\mathrm{AB})$ to quantify the Quercetin release from the nanometric PCL/ $\mathrm{Q}$ films known its high sensitivity and reliable response to the amount of mass adsorbed on the sensor surface. ${ }^{22}$ Prior to their use in the experiments, QSX303 Silicon coated quartz chips (QSense, Biolin Scientific $A B$ ) were sonicated in ethanol for 20 min, dried under $\mathrm{N}_{2}$ stream, and treated with UV/ozone for $30 \mathrm{~min}$. Sensors were then spin coated pouring two drops at $3600 \mathrm{rpm}$ of the $10 \mathrm{mg} \mathrm{mL}{ }^{-1} \mathrm{PCL} / \mathrm{Q}$ solution before being mounted into the QCM-D chamber. Experiments were performed at $37{ }^{\circ} \mathrm{C}$ and the volume of nitrogen-treated PBS contained in each chamber was $300 \mu \mathrm{L}$. Real time variations of frequency $(\Delta f)$ and dissipation $(\Delta D)$ parameters were observed at several overtones $(n=3,5,7, \ldots, 13)$ throughout the QCM-D experiment. Complementarily, $D$ vs. $f$ plots ( $D f$ plots) were also depicted. Each point in these $D f$ plots represents a dissipation and frequency data at a certain time, which provides a more detailed view on the viscoelastic evolution of films per mass unit $(\Delta m)$ change. Hence, we used the Sauerbrey equation $(\Delta m=$ $-C \Delta \phi$ ) for correlating variations in the oscillation frequency of the quartz crystal with mass deposition/desorption processes. The analysis of the mass loss during the experiment enables the study of Quercetin release kinetics from PCL nanometric films both with and without the S-layer coating. Then, since the polymer did not degrade during the experiment, ${ }^{23}$ the mass loss is only attributable to the release of Quercetin.

\section{Cell proliferation}

In order to assess cellular affinity for this type of PCL/Q blends, both with and without a S-layer coating on top, cell proliferation experiments were performed. In this regard, Human Umbilical Vessel Endothelial cells (HUVEC) were grown in T75 flasks using high glucose Dulbecco's modified eagle medium with stable glutamine, supplemented with $10 \%$ fetal bovine serum and $1 \%$ penicillin/streptomycin. Cells were cultured at $37{ }^{\circ} \mathrm{C}$ with $5 \% \mathrm{CO}_{2}$. Prior to microscopy experiments, cells were trypsinized using $2 \mathrm{~mL}$ TrypLETM Express, centrifuged and counted. Borosilicate circular cover glasses (diameter: $24 \mathrm{~mm}$, thickness: 0.08-0.12 mm, Menzel Gläser, VWR, Germany) were rinsed with EtOH, $\mathrm{N}_{2}$ dried and cleaned with oxygen plasma (GaLa Instrumente $\mathrm{GmbH}$, Austria). Then, slides were coated with the corresponding film, as explained above. The PCL or PCL/Q-coated slides were then incubated for $24 \mathrm{~h}$ in the presence of $4 \times 10^{4}$ cells suspended in DMEM. Prior to measurements, the medium was changed to Leibovitz's L-15. Cell analysis was then performed using a Axio Observer Z1 (Zeiss $\mathrm{GmbH}$, Jena, Germany) inverse optical microscope, with a $20 \times$ objective. Micrographs were processed with the ZEN Imaging Software (Zeiss GmbH, Jena, Germany), which allowed statistical estimation of the cell body area under each experimental condition.

\section{Results and discussion}

\section{Crystallization behavior based on the X-ray diffraction analysis}

The XRD patterns of PCL, Quercetin and their blends are shown in Fig. 1. Quercetin exhibits a characteristic intense peak at $2 \theta=13^{\circ}$, as well as several strong peaks at $11^{\circ}, 14^{\circ}$ and $28^{\circ}$. As it was predicted through DSC results (see Fig. S2 of the ESI $\dagger$ ), owing to the strong crystalline nature of the Quercetin, when there is more than $20 \mathrm{wt} \%$ of Quercetin in the blend, it is able to auto-associate and form crystals. Thus, small peaks at $11^{\circ}, 13^{\circ}$ and $28^{\circ}$ appeared in XRD PCL/Q 60/40 plot. Fortunately, polymer chains of the PCL are able to maintain the highly crystalline flavonoid in an amorphous state due to the intermolecular interactions, and this could be clearly seen in the PCL/CAM 90/10 blend, in which crystallization of Quercetin is completely suppressed. Hence, completely amorphous Quercetin (as required in amorphous solid dispersions, ASDs) can only be obtained when the Q content is below $20 \mathrm{wt} \%$.

\section{In situ monitoring of bacterial S-layer protein self-assembly on PCL/Q nanometric films}

The real-time assembly of SbpA proteins on top of PCL-based samples could be monitored by means of QCM-D experiments (Fig. 2). For such a purpose samples composed of either $10 \%$ or $20 \%$ Quercetin were employed. The drug content of interest was 


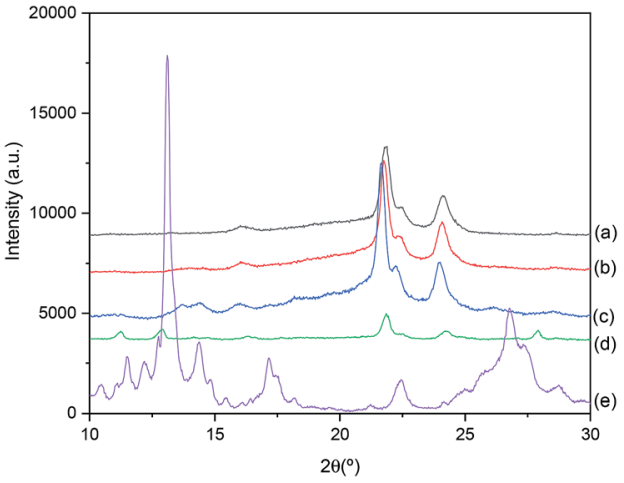

Fig. 1 XRD patterns of: (a) pure PCL; (b) PCL/Q 90/10; (c) PCL/Q 80/ 20; (d) PCL/Q 60/40; and (e) pure Q.

decided based on two main factors: drug solubility and the toxicity of the final mixture, being both optimal enough in the two compositions chosen. Indeed, these two blend compositions still maintained the classical spherulite-featured appearance of pure PCL films, as determined by AFM imaging (see Fig. S5 of the ESI $\dagger$ ).

Upon injection of $100 \mu \mathrm{g} \mathrm{mL}{ }^{-1}$ SbpA in divalent $\mathrm{Ca}^{2+}$ containing crystallization buffer, an immediate variation in both frequency and dissipation factors was observed. These parameters correlate, respectively, to the SbpA mass being adsorbed at the interface and the variation of the film (visco)elasticity. Hence, only after five minutes of incubation, the achievement of a plateau in frequency $(\Delta f)$ around $-100 \mathrm{~Hz}$ indicated the completion of the SbpA adsorption, which tells about an extremely fast process as previously observed for substrates of similar nature to that of PCL. ${ }^{24-26}$ Simultaneously, $\Delta D$ values present a constant evolution featured by a first increase, the subsequent appearance of phase transition peak typically featuring S-layer formation on hydrophobic substrates, and a drop to lower values. ${ }^{16}$ Such a transition is very clear when $\Delta D$ is plotted $v s . \Delta f$, in a so-called $D f$ graph, as depicted in Fig. 2b. On it, the decreasing $D$ values derive from re-arrangement of the protein film towards a crystal-like coating as more mass is incorporated, which finally rendered a structure like the one shown in Fig. 2c. This AFM height micrograph shows the resulting square (p4) lattice of the S-layer, confirmed by the Fast Fourier Transform analysis in the inset.

From these results it can be observed how the SbpA assembly proceeds very efficiently on both samples and showing an overall resemblance, independently from the Quercetin content. The most relevant variation between them, if going very deep in details, would come from the way that dissipation values evolve. A higher content of the drug seems to affect the re-arrangement of the crystalline film in terms of time to equilibrium. This is, a larger amount of Quercetin might influence the motility of the protein subunits either because of a higher lateral fluidity of the PCL film underneath or because the shift in wettability it causes. In both cases the final S-layer structure obtained was identical.

\section{Quantification of the S-layer influence on the Quercetin release}

In vitro release experiments were performed for PCL/Q 90/10 and 80/20 films where the Quercetin is in amorphous form, ready to be released by diffusion. The first step of such a release is the penetration of the buffered solution into the polymeric matrix, thus leading to film swelling. As a result, the polymeric network becomes looser, and the amorphous Quercetin dissolved within the polymer diffuses throughout it to reach the outer solution. ${ }^{27}$ As aforementioned, and due to the heterogeneity of the samples prepared (thick $v s$. thin a)

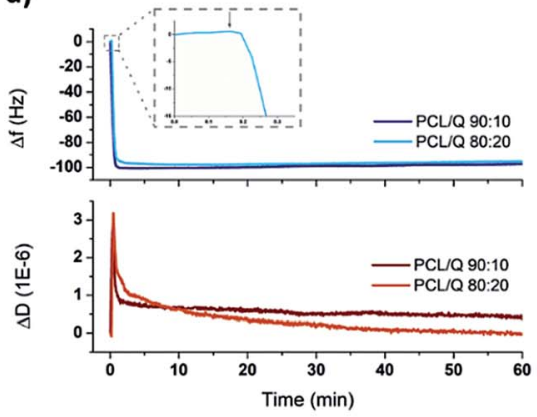

b)

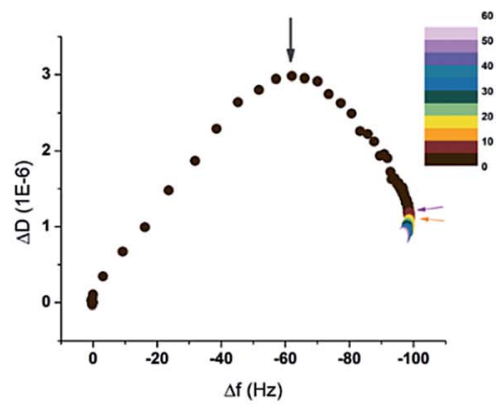

c)

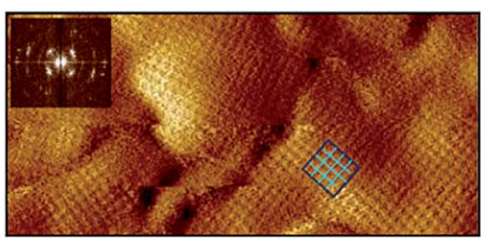

Fig. 2 (a) Real time QCM-D monitoring of the SbpA S-layer formation on top of PCL/Q blends. The inset magnifies the first minutes after the protein injection, as indicated by the grey arrow in it. (b) Resulting Df plot for the first 60 min of process observation. The black arrow points the moment at which transition occurs. The colorscale reflects the time elapsed after SbpA injection. (c) AFM height micrograph of the S-layer topography on top of an underlying PCL-derivative film. The inset show the applied fast Fourier transform with a clear square ( $\mathrm{p} 4)$ repetition unit being observed. A drawing highlights the type of structures assembled. 


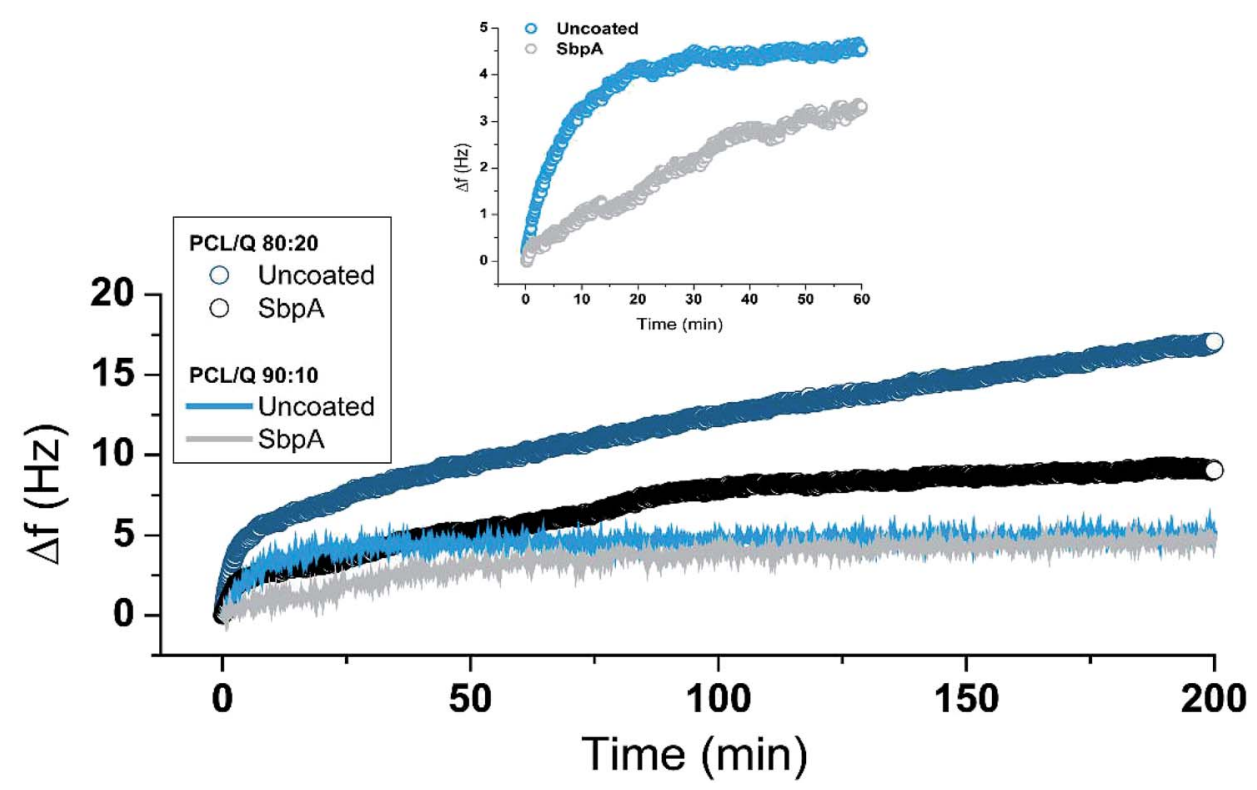

Fig. 3 Real time monitoring of the Quercetin release from PCL/Q blends either with or without S-layer coating on top. The inset magnifies the first 60 minutes of the release as measured for $90 / 10$ samples.

films, 2D vs. 3D), the monitoring of the drug release was performed by different - though complementary methodologies.

Nanometric films. The influence of applying a S-layer coating on the release of Quercetin from thin (200 nm) PCL/Q blends was quantified by means of QCM-D technique. Environmental conditions were adapted to ensure an optimal release rate (degassed PBS and $37{ }^{\circ} \mathrm{C}$ ) over the entire experiments, performed in parallel for both 90/10 and 80/20 blend compositions, as shown in Fig. 3.

At first glimpse, uncoated PCL/Q 80/20 systems showed the largest drug release (expressed by larger positive frequency variations, around $+17 \mathrm{~Hz} v s$. $+5 \mathrm{~Hz}$ ) because of the higher initial content. However, the presence of the SbpA coating successfully slowed down the release process, even from the very initial moments. After the initial release peak, which occurs in both samples, the Quercetin release proceeds over the time following an almost linear (zero order) release trend. From the slope values of such linear fitting, a 3-fold decrease is calculated for the release in the presence of an S-layer membrane. A similar behavior was recorded for PCL/Q 90/10 composition, although the total release is constrained by the spherulitic morphology of the blend (Fig. S5 $\dagger$ ). In this case, the presence of the S-layer offered a significant attenuation of the initial Quercetin being released, as highlighted in the inset from Fig. 3.

In both compositions, the S-layer slows down the initial burst allowing a zero order release mechanism (constant rate) that is desirable in order to minimize oscillations in the drug concentration in blood, which could induce episodes of toxicity. ${ }^{28}$

Micrometric films. In the case of the micrometric films ( $c a$. $200 \mu \mathrm{m}$ ), the S-layer was formed by immersion of a $1 \mathrm{~cm}^{2}$ piece into SbpA protein containing recrystallization buffer. Because of the temperature control exerted, spontaneous drug release over exposure to SbpA could be effectively neutralized. This was certified by the absence of the Quercetin featuring peak in the spectrogram obtained from the protein solution at the end of the incubation process.

As can be seen in Fig. 4, the release of Quercetin from thick PCL/Q films with an outer S-layer lasted longer and proceeded smoother if compared to their uncoated partners. Considering the release from both $80 / 20$ and 90/10 compositions after the first $60 \mathrm{~min}$ of the experiment, the release could be restrained a $30 \mathrm{wt} \%$ with the help of the S-layer coating. Furthermore, as occurred in the case of the nanometric platforms, the slope of the release plot underwent a 3-fold decrease with the S-layer in a linear fitting or zero order kinetics, useful for sustained drug delivery systems.

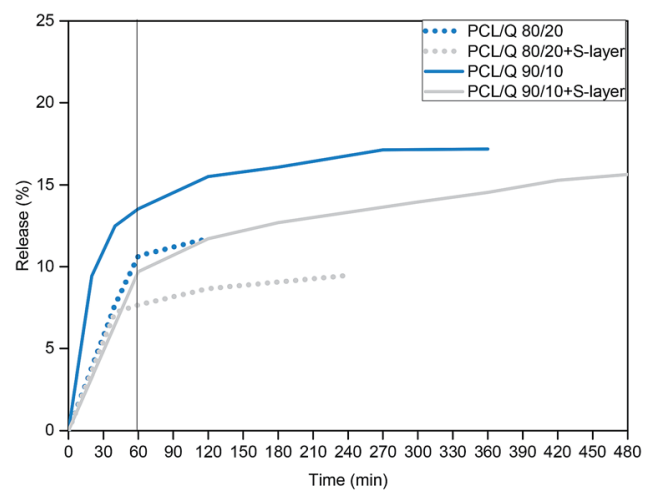

Fig. 4 Drug release profile of $P C L / Q 80 / 20$ and 90/10 samples with a thickness of $200 \mu \mathrm{m}$ obtained by solvent casting, immersed into $50 \mathrm{~mL}$ of nitrogen treated-PBS $\left(\mathrm{pH} \mathrm{7.4)}\right.$ at $37^{\circ} \mathrm{C}$ during 8 hours. The vertical line located at the first $60 \mathrm{~min}$ helps to display the great decrease found in the release with the presence of the $\mathrm{S}$-layer coating. 


\section{Effect of the 3D structure on the Quercetin release}

Having a good control over drug delivery processes in electrospun fibers turns also very promising due to their large specific surface area, which results in a high drug-loading capacity. Other appealing features for electrospinning in this field include high encapsulation efficiency, the possibility of simultaneous delivery of diverse therapies, ease of operation, as well as cost-effectiveness. ${ }^{29-31}$ From the first study made by Kenawy et al. in which nanofibers were successfully applied as drug delivery systems, ${ }^{32}$ different controlled drug release profiles, such as immediate, smooth, pulsatile, delayed, and biphasic releases, have been successfully developed..$^{33,34}$

In this section, the burst release of Quercetin was studied in two different PCL platforms (a film and a mat made by electrospinning) with similar thickness $(\sim 125 \mu \mathrm{m})$, and both having a flavonoid content of wt $10 \%$. It is well known that the structural properties (surface area to volume ratio and porosity, along with the thickness) of the release platform, undoubtedly affect the release behavior of the drug. ${ }^{35}$ Therefore, tunable release profiles can be achieved by simply altering the physical morphology of the PCL-Quercetin systems. During the duration of this study, PCL did not degrade and, therefore, the measured drug can merely be attributed to Quercetin diffusion from both the matrix and the porous structures.

As shown in Fig. 5, different release profiles were obtained depending on the type of platform, concluding that the release rate of Quercetin increased with the larger presence of pores in the nanofibers mat. This is explained by the greater number of interactions between the eluting medium and the material. Contrarily, the non-porous 2D film displayed lower water uptake capacity and, the release only reached a $15.5 \%$ in the first 2 hours with a final percentage of $17.2 \%$ at the end of the study in the absence of S-layer. After SbpA coating these percentages dropped to $11.71 \%$ ( 2 hours) and $15.6 \%$ at the end. In the case of $3 \mathrm{D}$ platforms, after 2 hours of experiment, the nanofibers mat with the S-layer showed a release of $22 \%$, while the scaffold without the membrane had already released the $31.1 \%$, which, again, represents a reduction of the $30 \%$.

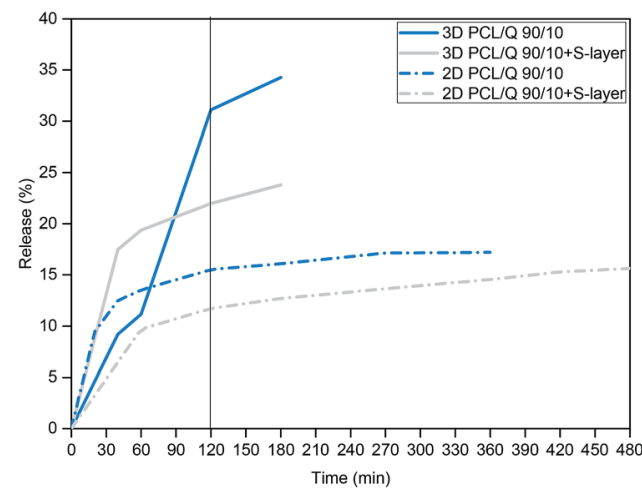

Fig. 5 Quercetin release profiles of PCL/Q 90/10 samples of different structures with a thickness of $125 \mu \mathrm{m}$, immersed into $50 \mathrm{~mL}$ of nitrogen treated-PBS at $37^{\circ} \mathrm{C}$ for $8 \mathrm{~h}$. The vertical black line represents the first 2 hours of the experiment when the initial burst has already occurred.
According to electron microscopy images (Fig. 6), employed for testing potential superficial variations along the release process, 2D films lost their superficial crystalline structure during the time they stayed submerged in PBS buffer solution. Furthermore, due to the combined effect of drug diffusion together with the erosion caused by the surrounding medium, the appearance of micro-sized pores could be observed (see Fig. 6a). In contrast, electrospun scaffolds maintained its fibrous structure throughout the entire experiment (Fig. 6b), although slight overall structural variations resulted from their immersion.

\section{Cell proliferation experiments}

Complementarily to the characterization above, cell proliferation studies were also performed. Endothelial HUVEC cells were seeded on top of the aforementioned systems (pure PCL, PCL/Q $90 / 10$ and $80 / 20$ films with a thickness of $200 \mathrm{~nm}$ ), and the resulting cell area was measured after 24 hours of incubation under appropriate conditions $\left(\mathrm{CO}_{2}\right.$ atmosphere, cell growth medium). Measurement of the cell area is considered an optimal factor to characterize the cell-substrate affinity, since the larger it results the better cells can freely spread over the composition underneath. ${ }^{36}$ Calculated results from optical microscopy images, as well as the derived statistical analysis, are shown in Fig. 7.

According to the observations done, HUVEC cells on pure PCL are capable of spreading to a certainly quite larger extent than those on Quercetin-containing systems, which do not reflect significant differences in terms of drug content. Khampieng et al. recently described the potential influence of the topography of PCL films on cell attachment. ${ }^{37}$

Mean cell area values are collected in Table 1 for a better visualization of the numerical changes. The box on top of the

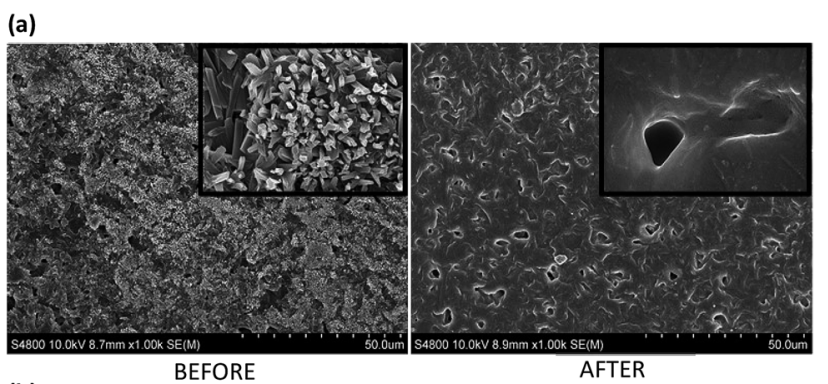

(b)

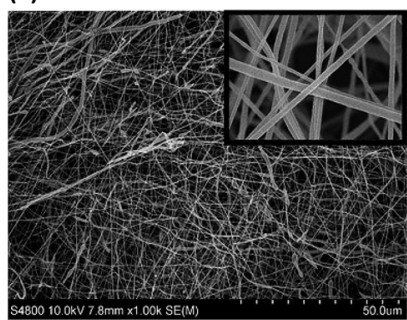

BEFORE

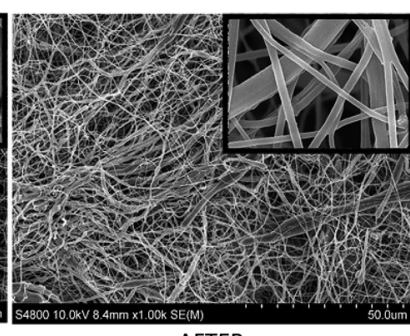

AFTER
Fig. 6 FE-SEM images of PCL/Q 90/10: (a) film, and (b) nanofibers scaffold, before and after 8 hours of in vitro Quercetin release (zoom areas corresponds to a magnification of $20000 \times$ ). 


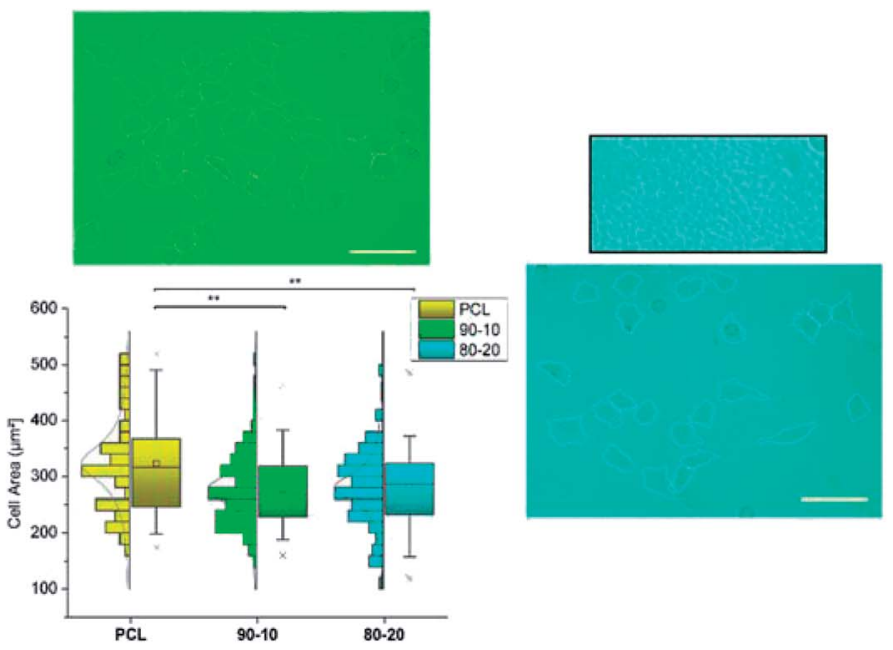

Fig. 7 Cell area calculation from the corresponding optical micrographs on the different PCL systems. Statistical analysis was performed for $N>$ 100 and a one-way ANOVA determined the significance $(p<0.01$, represented by "**") of the recorded variations.

Table 1 Mean HUVEC body area values after 24 hours of incubation on the corresponding substrate

\begin{tabular}{ll}
\hline Sample & Mean cell area $\left(\mu \mathrm{m}^{2}\right) \pm \mathrm{SE}$ \\
\hline PCL & $323.4 \pm 11.8$ \\
PCL/Q 90/10 & $277.8 \pm 5.8$ \\
PCL/Q 80/20 & $280.9 \pm 6.5$ \\
\hline
\end{tabular}

$80 / 20$ case shows a magnified view of the background, with the clear presence of features that do not appear in those cases where PCL crystallinity is maintained. These results support the choice of 90/10 and 80/20 PCL/Q compositions, as they remain below the critical toxicity levels.

\section{Anti-fouling activity of SbpA S-layer coating}

The presence of the SbpA S-layer does not only provide the PCL/ $\mathrm{Q}$ blends with an effective release-controlling membrane but also endows these films with anti-fouling properties, known the good non-fouling activity of such type of coatings. ${ }^{12,13}$ Continuing with the methodology explained in the previous section, HUVEC cells were seeded on different PCL/Q ratios which had previously been employed for the recrystallization of SbpA bacterial proteins. Again, samples were kept for 24 hours under cell growth promoting conditions and the cell area was measured as shown in Fig. 8.

The first striking result regarded the number of individual cells found in direct contact with the surfaces, which is
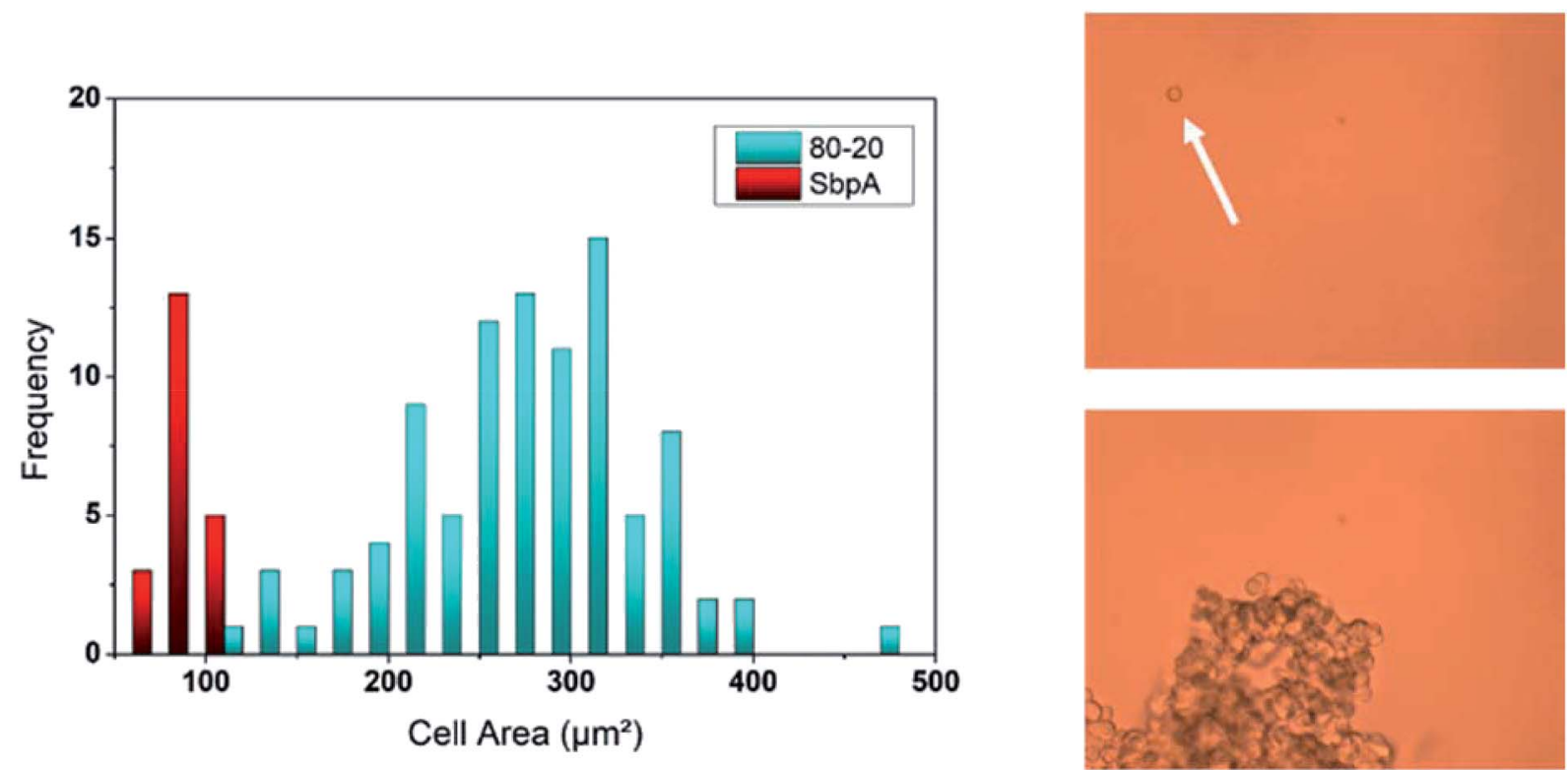

Fig. 8 Histogram showing the cell area distribution for PCL/Q 80/20 systems with and without S-layer coating. Pictures on the right show the case of an individual HUVEC cell on top of the S-layer, and an example of a usual cell aggregate. 
minimum. Instead, HUVEC cells are observed to float over the substrate because of the lack of cell-substrate interactions. Cells prefer forming tissue-like aggregates of diverse sizes with other floating HUVECs, as already reported in literature by MorenoFlores et $a .^{38}$ attending to the cell area measured those cells on top of SbpA are found to be around 70-90 $\mu^{2}$, corresponding to an almost perfect spherical shape. Such values represent four-fold decrease in the observed HUVECs area than those on top of SbpA-less films, which speaks about the effectiveness of the applied coating.

\section{Conclusions}

The preparation of Amorphous Solid Dispersions (ASDs) based on PCL and Quercetin mixes appears as a very promising synthetic drug delivery system for biomedical applications. However, despite the healthiness of Quercetin - attending to its proven antioxidant and anti-inflammatory properties-, this flavonoid possesses a high crystallinity, low bioavailability and potential toxicity that might compromise its therapeutic use.

In this regard, it was first found that PCL/Q blends are miscible (monophasic), and that for Quercetin weight compositions below $20 \%$ crystallization is effectively prevented. Consequently, PCL/Q 80/20 and 90/10 compositions have been employed for investigations regarding their release performance and their activity. This could also be supported by the topography analysis performed by AFM indicating the maintenance of the featuring spherulite appearance of PCL.

Second, and in order to minimize the potential toxicity of the Quercetin, a nanometric S-layer coating from SpbA bacterial proteins has been applied on top of the PCL/Q specimens to help regulate the drug release. SbpA protein subunits have the capacity of reassembling by following a square lattice with welldefined nano-pores, giving thus raise to a permeable membrane with proven anti-fouling properties. The influence of presence of the S-layer on the drug release mechanism has been tested for both 2D films of diverse thickness and 3D platforms (fiber mats). Hence, a stable linear release was obtained (30\% decrease compared to uncoated samples) in all the cases. The faster release from such 3D platforms can be explained by the bigger specific contact area, as the molecules have more space for drug diffusion.

Complementary cell viability (HUVECs) studies on SbpA-free PCL/Q films have been employed to show the lack of toxicity of the films after $24 \mathrm{~h}$ of incubation, and the impressive nonfouling performance of those containing an S-layer on top, which prevents the blocking of the membrane activity. This property turns of particular interest for the avoidance of bacterial colonization or binding of other foulants, which could compromise the use of these materials for medical purposes.

\section{Conflicts of interest}

There are no conflicts to declare.

\section{Acknowledgements}

The authors are thankful for funds from the Spanish Ministry of Innovation and Competitiveness MINECO (MAT2016-78527-P), the Basque Government, Department of Education (IT-927-16) and the EU iPROMEDAI COST Action TD1305. Authors also want to thank Jacqueline Friedmann and Amsatou AndorferSarr for technical support with the SbpA purification and cell culture.

\section{Notes and references}

1 A. Panche, A. D. Diwan and S. R. Chandra, J. Nutr. Sci., 2016, $5,1$.

2 S. Kumar and A. K. Pandey, Sci. World J., 2013, 162750S.

3 D. Aune, E. Giovannucci, P. Boffetta, L. T. Fadnes, N. Keum, T. Norat, D. C. Greenwood, E. Riboli, L. J. Vatten and S. Tonstad, Int. J. Epidemiol., 2017, 46, 1029.

4 F. Puoci, C. Morelli, G. Cirillo, M. Curcio, O. I. Parisi, P. Maris, D. Sisci and N. Picci, Anticancer Res., 2012, 32, 2843. 5 Y. Guo and R. S. Brun, J. Nutr. Biochem., 2015, 26, 201.

6 V. Natarajan, N. Krithica, B. Madhan and K. Sehgal, J. Pharm. Sci., 2011, 1, 195.

7 R. Chen, J. Lin, J. Hong, D. Han, A. D. Zhang, R. Lan, L. Fu, Z. Wu, J. Lin, W. Zhang, Z. Wang, W. Chen, C. Chen and H. Zhang, Toxicol. Rep., 2014, 1, 450.

8 M. Labet and W. Thielemans, Chem. Soc. Rev., 2009, 38, 3484. 9 N. Shah, H. Sandhu, D. S. Choi, H. Chokshi and A. Malick, Amorphous Solid Dispersions: Theory and Practice, Springer, NY, 2014.

10 A. Robaszkiewicz, A. Balcerczyk and G. Bartosz, Cell Biol. Int., 2007, 31, 1245.

11 U. B. Sleytr, B. Schuster, E. Egelseer and D. Pum, FEMS Microbiol. Rev., 2014, 38, 823.

$12 \mathrm{~J}$. Iturri, A. Moreno-Cencerrado and J. L. Toca-Herrera, Colloids Surf., B, 2017, 158, 270.

13 A. Moreno-Cencerrado, J. Iturri, I. Pecorari, M. Vivanco, O. Sbaizero and J. L. Toca-Herrera, Microsc. Res. Tech., 2017, 80, 124.

14 S. Chung, S. H. Shin, C. R. Bertozzi and J. J. De Yoreo, Proc. Natl. Acad. Sci. U. S. A., 2010, 38, 16536.

15 A. Breitwieser, J. Iturri, J. L. Toca-Herrera, U. B. Sleytr and D. Pum, Int. J. Mol. Sci., 2017, 18, 400.

16 J. Iturri, A. C. Vianna-Cintra, A. Moreno-Cencerrado, D. Pum, U. B. Sleytr and J. L. Toca-Herrera, Beilstein J. Nanotechnol., 2017, 8, 91.

17 A. Moreno-Cencerrado, J. Iturri and J. L. Toca-Herrera, Microsc. Res. Tech., 2018, 81, 1095.

18 J. Iturri, A. Moreno-Cencerrado and J. L. Toca-Herrera, Coatings, 2019, 2, 76.

19 U. B. Sleytr, M. Sára, Z. Küpcü and P. Messner, Arch. Microbiol., 1986, 1, 19.

20 M. Bancirova, Chem. J., 2015, 1, 31.

21 A. Seidell and D. Van, Solubilities of Organic Compounds, Norstrand Company, NY, 1941.

22 G. Celik and A. U. Oksuz, J. Macromol. Sci., Part A: Pure Appl. Chem., 2015, 52, 76. 
23 J. Fernández, A. Etxeberria and J. R. Sarasua, Eur. Polym. J., 2015, 71, 585.

24 A. Lejardi, A. E. López, J. R. Sarasua, U. B. Sleytr and J. L. Toca-Herrera, J. Chem. Phys., 2013, 139, 121903.

25 J. L. Toca-Herrera, R. Krastev, V. Bosio, S. Küpcü, D. Pum, A. Fery, M. Sára and U. B. Sleytr, Small, 2005, 1, 339.

26 J. Iturri, A. Moreno-Cencerrado and J. L. Toca-Herrera, Colloids Surf., A, 2017, 526, 56.

27 J. Siepmann and N. A. Peppas, Adv. Drug Delivery Rev., 2001, 48, 139.

28 R. A. Siegel and M. J. Rathbone, Fundamentals and Applications of Controlled Release Drug Delivery, Springer, US, 2012.

29 X. Hu, S. Liu, G. Zhou, Y. Huang, Z. Xie and X. Jing, J. Controlled Release, 2014, 185, 12.

30 B. Wang, Y. Wang, T. Yin and Q. Yu, Chem. Eng. Commun., 2010, 197, 1315.
31 S. Chakraborty, I. C. Liao, A. Adler and K. W. Leong, Adv. Drug Delivery Rev., 2009, 61, 1043.

32 E. R. Kenawy, G. L. Bowlin, K. Mansfield, J. Layman, D. G. Simpson, E. H. Sanders and G. E. Wnek, J. Controlled Release, 2002, 81, 57.

33 M. Prabaharan, R. Jayakumar and S. V. Nair, Adv. Polym. Sci., 2012, 246, 241.

34 Y. Z. Zhang, C. T. Lim, S. Ramakrishna and Z. M. Huang, J. Mater. Sci.: Mater. Med., 2005, 16, 933.

35 A. Laha, S. Yadav, S. Majumdar and C. S. Sharma, Biochem. Eng. J., 2016, 105, 481.

36 M. Sumarokova, J. Iturri, A. Weber, M. Maares, C. Keil, H. Haase and J. L. Toca-Herrera, Sci. Rep., 2018, 8, 9660.

37 T. Khampieng, V. Yamassatien, P. Ekabutr, P. Pavasant and P. Supaphol, Adv. Polym. Technol., 2018, 37, 2030.

38 S. Moreno-Flores and S. Küpcü, Soft Matter, 2015, 11, 1259. 